\title{
Sensor Comparison for a Real-Time SLAM Application
}

\author{
M. Korkmaz, A. Durdu, and Y. E. Tusun
}

\begin{abstract}
Different types of sensors are used for Simultaneous Localization and Mapping (SLAM) applications. These sensors have their own advantages and disadvantages. Although high accuracy rates, laser sensors have some disadvantages such as price, power requirements, and weight. As an alternatively, it is possible to use of inexpensive sensors such as Kinect which gives image and depth data in SLAM systems. There have been many studies that benefit from such sensors with good results and many of them are carried out in a simulation environment. However, there are few studies on whether similar outcomes will be valid for real-time applications. With this thought, a real-time application has been performed for comparison of both sensors in SLAM systems. In the light of obtained findings, this type of sensors is not a good alternative to laser sensors in terms of both map accuracy and time consumption.
\end{abstract}

Index Terms - Gmapping, SLAM, kinect, rplidar.

\section{INTRODUCTION}

Mapping and localization problems are a very important phenomenon for robotic systems [1], [2]. Depending on the solution of these two cases, a robot is able to have information about what the world around its looks like and several tasks might be ordered to the robot. In circumstances where GPS data are available, localization is not a matter and it is easy to build the map of the environment with known agent's locations. However, in some situations, it might not be possible to get GPS data. The most obvious example of such cases is the indoor applications that location data cannot be acquired with GPS sensors [3]. Taking consideration such situations, localization and mapping problems have to handle together and robot's location and the environment map should be generated simultaneously. This problem is known as Simultaneous Localization and Mapping (SLAM) in the literature [2].

One of the most difficult things on solving SLAM problem is the dependency between location and map. The robot needs location information while building the environment map and the robot localizes itself within the created map. All these processes are done by the sensors but no such a sensor has an excellent accuracy but has noise which will be important if the processes are repeated many times. This repetitive procedure causes to a cumulative error. At this point, it has been improved many algorithms to solve mentioned problems [2], [4]-[7].

In order to solve the SLAM problem, odometry data is usually utilized as an internal sensor while more sensor type can be used as an external sensor. Sonar sensors are drawn

\footnotetext{
Manuscript received December 29, 2017; revised March 10, 2018. This work was supported by Selcuk University Scientific Research Project Fund, BAP.

The authors are with the Electrical Engineering Department, University of Selcuk, Konya 42075, Turkey (e-mail: mkorkmazeem@gmail.com, durdu.1@selcuk.edu.tr, yunusemretusun@gmail.com).
}

upon in the first years to solve the problem, and the following periods, it is frequently seen that laser and camera sensors are more appropriate for external receptors [5], [8]-[9].

In this study, the question of how the effectiveness of the maps obtained with the use of different types of sensors is sought. In this context, the comparison of the RGB-D sensors [10] with the classical laser sensors and the efficiency of the produced maps is compared with a real-time study.

The second part of the article mentions the SLAM problem and compared sensors. The third part is about how the application is implemented and an environment is created. In the last part, the findings are discussed and it is emphasized what kind of studies should be done in the future periods.

\section{Slam AND UsED SENSORS}

\section{A. SLAM}

SLAM problem is thought as in the base of probabilistic view in equation 1 [3].

$$
P\left(X_{k}, m \mid Z_{k}, U_{k}\right)
$$

The motion and observation models are described within this framework (Eq. 2 and 3).

$$
\begin{gathered}
P\left(X_{k} \mid X_{k-1}, U_{k}\right) \\
P\left(Z_{k} \mid X_{k}, m\right)
\end{gathered}
$$

In the first period of the problem, solutions are focused on the use of Bayes based filters. At this stage, Extended Kalman Filter (EKF) is a milestone and significant progress has been recorded with this algorithm [2], [5]. However, the process time increases as quadratic depending on the number of landmarks and the solution is impossible when the environment has a big size. As a solution to this problem, particle filter based methods have been improved. The combination of the particle filter (PF) with Rao-Blackwellizied decomposition method allows to arise a series of the new algorithms such as FastSLAM [6], [7].

The biggest achievements of such methods are that time consumption decreases significantly and possible to use for non-Gaussian noises.

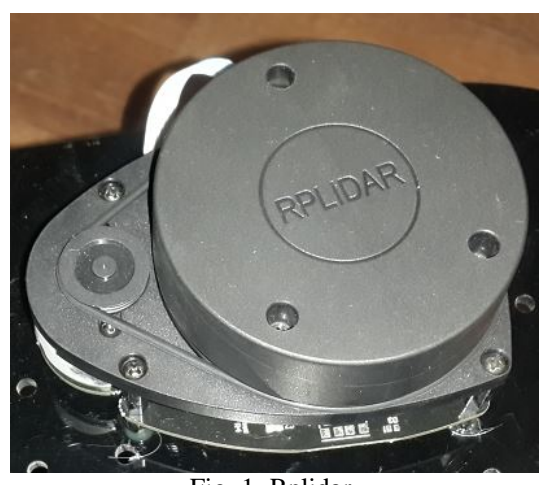

Fig. 1. Rplidar. 


\section{A. Rplidar}

The RPLidar (Fig. 1) scanner has a data acquisition capability up to 6 meters with 360 degrees rotation. It can collect data from the environment in the form of a point cloud cluster in a $2 \mathrm{D}$ plane. Generally, the speed is about $5.5 \mathrm{~Hz}$ but it can be increased up to $10 \mathrm{~Hz}$ when desired. This system, which is operated by laser triangulation measurement method, is a good alternative for applications such as indoor environment [11].

\section{B. Kinect}

The Kinect sensor is released by Microsoft in the year 2010 as a purpose of game console [12]. In the following years, companies such as Asus and Apple have launched similar sensors to market. These ones have attracted attention by robotic researchers because they can provide depth information about the environment as well as image features and have been used in many applications with different aims.

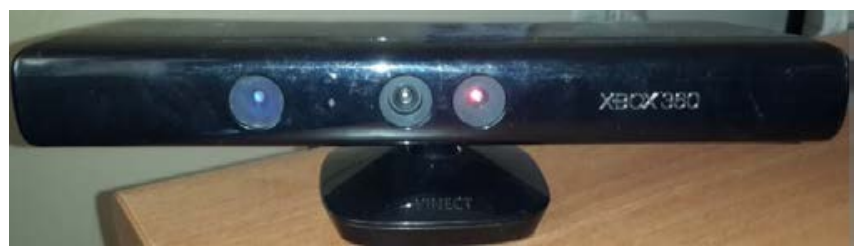

Fig. 2. Kinect.

This sensor has the ability of view 43 degrees vertical and 57 horizontal. It has been replaced by laser sensors in many applications providing depth image data that can be converted to point cloud and laser data.

Table I gives a comparison between RPLidar and Kinect with the angle of different features.

\begin{tabular}{|l|l|l|}
\multicolumn{2}{c}{ TABLE I: RPLIDAR VS. KINECT } \\
\hline & Rplidar & Kinect \\
\hline Price & $\sim 450 \$$ & $\sim 150 \$$ \\
\hline Range & $\sim 6$ meters & $\sim 5$ meters \\
\hline Angle & 360 degrees & 43 vertical, \\
& & 57 horizontal \\
& & degrees \\
\hline
\end{tabular}

\section{IMPLEMENTATION}

A comparison of sensors for SLAM systems has been dealt with in a real-time environment as in Fig. 3.

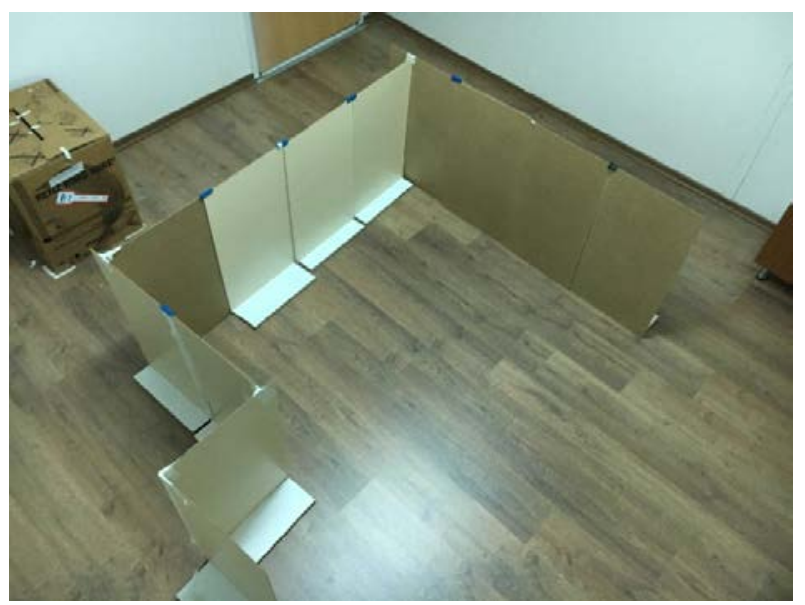

Fig. 3. The test environment.
This environment created under laboratory conditions is approximately $16 \mathrm{~m} 2$ and covered with corridors and obstacles at different measurements. An environment is designed in such a way that the map validity can be measured and the discontinuity points that can occur in the map can be seen better.

\section{A. Robot and Algorithm}

In order to carry out the experiments, it is needed a robot platform and with this aim, a robot known Turtlebot is used. This robot, known as Turtlebot-II, is often utilized in literature for preliminary studies and offers an easy and modular structure for attaching of different types of sensors. In particular, due to the compatibility with the Robot Operating System (ROS), it is frequently preferred by researchers and allows the use of different types of algorithms [13].

Traditional SLAM solutions are generally based on Bayes filters and its derivations. Although these algorithms can provide good solutions for small-size systems, the effectiveness of algorithms is reduced when worked in large-scale environments. This drawback forces the researchers to find a new way about large scale cases. As a result of studies, particle filter based methods are combined with the Rao-Blackwellization decomposition method and some kind of sequential algorithms called FastSLAM are produced.

The gmapping [7], [14] package on ROS platform, which is widely used in SLAM community, is performed to realize the experiments. This package is generally based upon FastSLAM and similar methods. It gives a grid map as an output where white areas mean free spaces that robot wanders, black areas are the obstacle, and gray spaces are uncertain. The logical levels of the mentioned territories are 255, 200, 0 respectively. The general flow chart for the mentioned algorithm is as follows (Fig. 4).

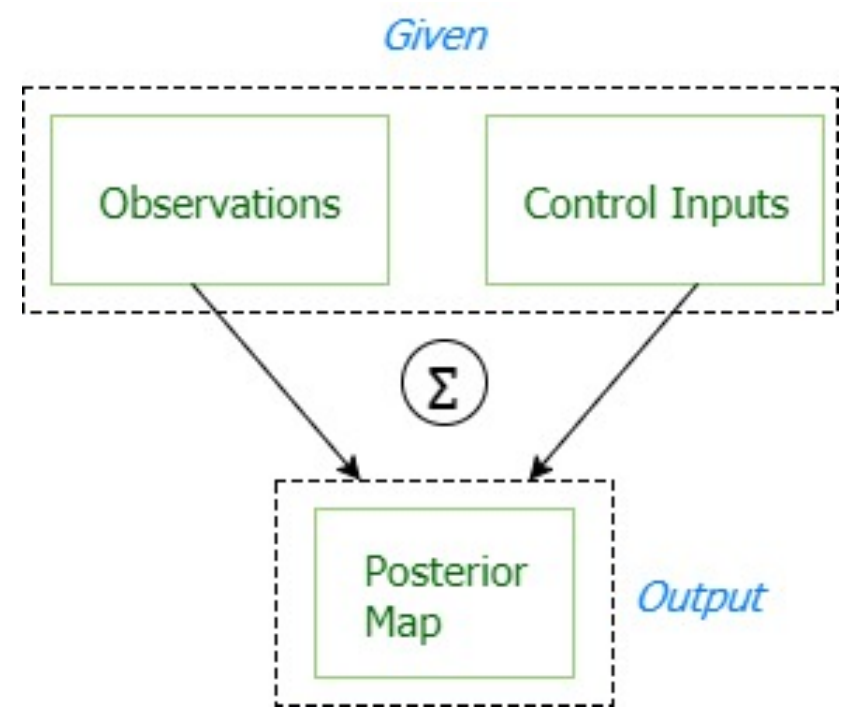

Fig. 4. Algorithm general sketch.

\section{B. Real-Time Application}

The Turtlebot robot and the sensors mentioned are used for mapping studies in an environment which aerial viewpoint are given in Fig. 5. In order to build the maps, the robot is navigated through the user guidance. 


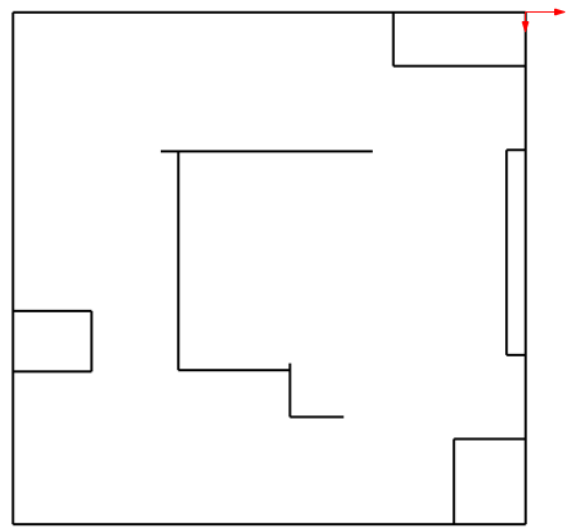

Fig. 5. Aerial viewpoint for test environment.

Firstly, Kinect sensor is coupled to the robot and the map of the environment is created as in Fig. 6 and 7. These maps are obtained under the several particle number and other parameters.

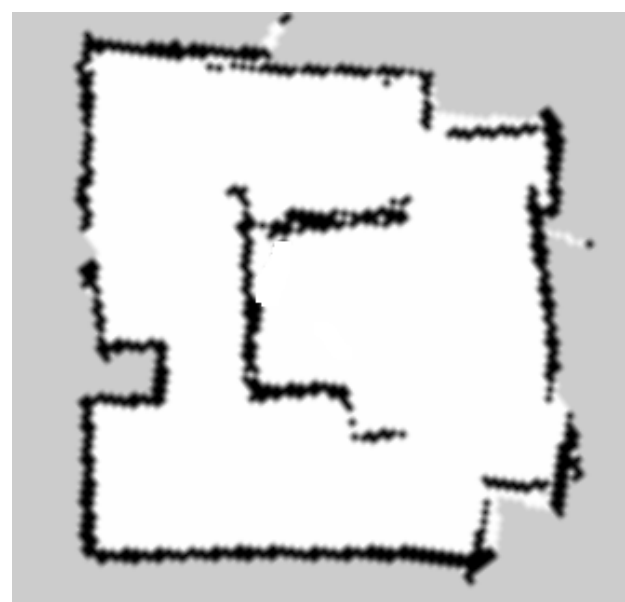

Fig. 6. Map-1 with kinect sensor, Particle number: 80.

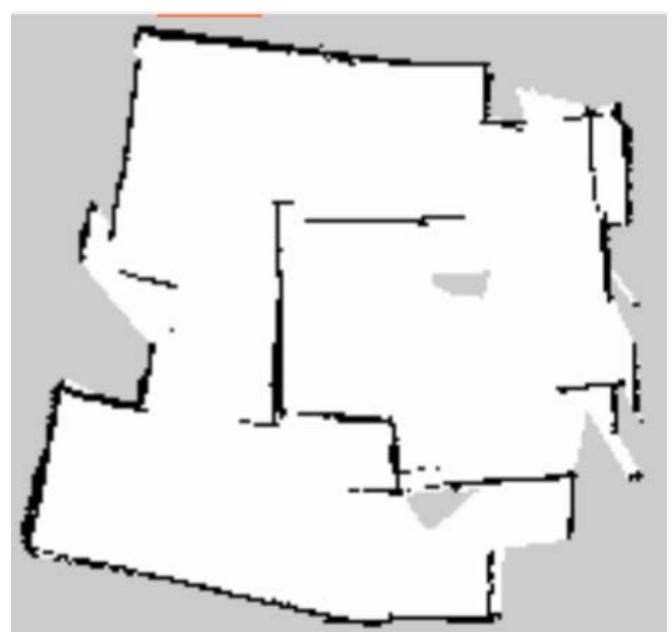

Fig. 7. Map-2 with kinect sensor, Particle number: 300.

Secondly, RPLidar is coupled to the robot and the maps obtained are in Fig. 8 and 9. Different parameters are set similar to Kinect sensor to create accurate maps.

It is clearly seen the effect of Kinect's sensor narrow-angle of view and its inactivity for the distances below 0.6 meters in the practices. Contrary to this, it is obvious the effect of the laser sensor high accurate and 360 degrees scan angle. The adjustment of the parameters is also vital issue to get effective and certain maps. These parameters sometimes are related to the environment and for this reason, the parameters have to be adjusted according to the environment with some tries.

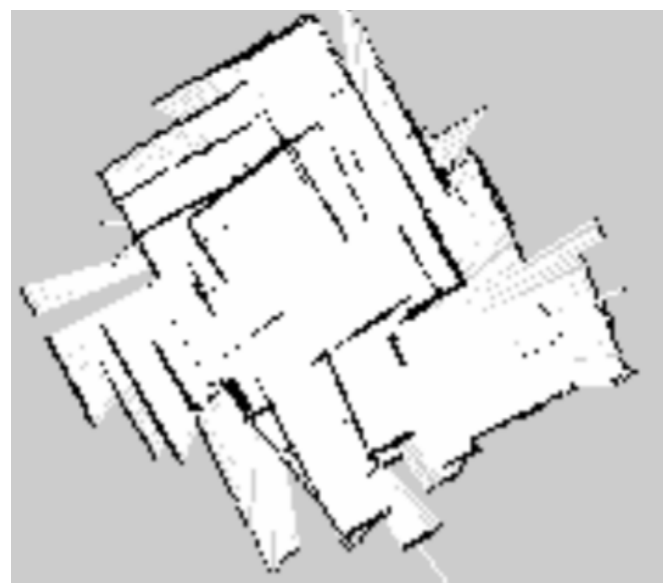

Fig. 8. Map-1 with RPLidar sensor, Particle number: 80 .

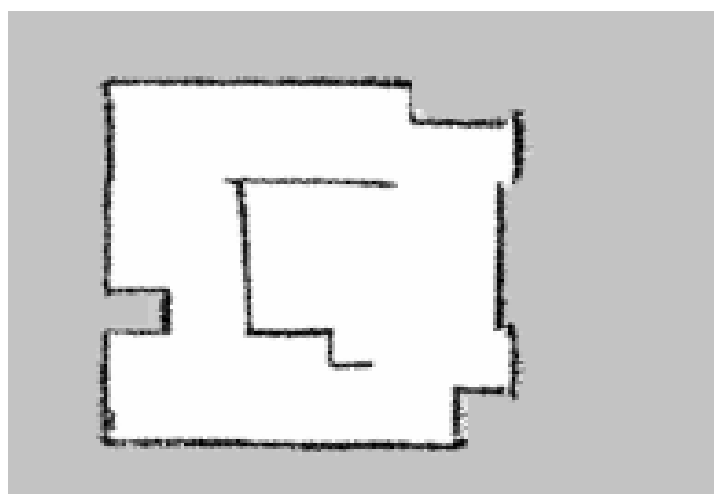

Fig. 9. Map-2 with RPLidar sensor, Particle number: 300.

Another prominent point in the results is the time spent for the whole process. Table II clearly shows the RPLidar time efficiency comparing the Kinect and the time spent with RPLidar is nearly one of three with the Kinect one. This result is directly related to the angle view and data intensity which RPLidar has more.

\begin{tabular}{|c|c|}
\hline TABLE II: ELAPSED TIME FOR EXPERIMENTS \\
\begin{tabular}{|c|c|}
\hline Sensor Type & Time (second) \\
\hline RPLidar & 92 \\
\hline Kinect & 210 \\
\hline
\end{tabular}
\end{tabular}

\section{REsults AND CONCLUSION}

Simultaneous Localization and Mapping is a topic that is frequently discussed in the literature in recent years. The development of such algorithms has made possible the autonomous movement and inspired many applications. The ability of such algorithms to produce good results is closely related to the sensors used. In this context, many studies have been done in the literature using different types of sensors.

The ability of the laser sensors to measure with high accuracy is suitable for SLAM systems. However, considering both the weights and the prices, these sensors may not be suitable for some systems, and this problem has 
led to a new kind of sensor searches. Recently, the different use of RGB-D cameras which has image and depth information has been examined by the researchers. By utilizing this extra depth feature, this sensor can be used as a laser sensor. Within this mind, this study investigates whether these sensors can be an alternative to laser sensors for a real-time application.

Fig. 5 points out the aerial view of the environment wanted to build the map, and Fig. 7 and 9 are the maps created with the sensors Kinect and RPLidar, respectively.

When the generated maps are examined, the map constituted by using RPLidar sensor has nearly exact matching to the test environment. On the contrary, the map obtained with the Kinect sensor has some serious defects.

As a result, in this study, the sensors used for SLAM systems are compared and the real-time performance of the sensors, which generally produce good results in the simulation environment, is measured comparatively. It is aimed to map the real-time environment so that the comparison can be done. Within this scope, mapping of an area of 16 square meters has been done. The information given by the Kinect sensor is used as laser data. It has been shown that this sensor cannot give proper results in real-time mappings due to the fact that the scanning angle is limited, and it does not provide a real laser data. Even if the properties of the algorithm such as the number of particles are increased, the result is not better. Conversely, the RPlidar laser scanner has shown effective results that are closer to real-world measurements with 360-degree rotation and true laser data.

It has been seen that RPLidar sensor has given good results both in time and map continuity. A Kinect-like sensor does not provide good results due to the fact that it has a wide angle.

When all these findings are addressed, it has been found that the work to be done using laser data for real-time applications of SLAM systems is closer to reliable and realistic environment measurements. The use of Kinect-like sensors as laser one may lead to the problems such as a discontinuity in the map and it does not reflect the real environment measurements. So this reason, it is highly likely to cause some problems when assigning a task to the robot.

It is aimed to compare the same and different sensors with other ROS packages and evaluate the results accordingly.

\section{ACKNOWLEDGMENT}

Authors are thankful to Rac-Lab (www.rac-lab.com) for providing the trial version of their commercial software for this study.

\section{REFERENCES}

[1] D. Droeschel, M. Schwarz, and S. Behnke, "Continuous mapping and localization for autonomous navigation in rough terrain using a 3D laser scanner," Robotics and Autonomous Systems, vol. 88, pp. 104-115, 2017.

[2] H. Durrant-Whyte and T. Bailey, "Simultaneous localization and mapping: part I,” IEEE Robotics \& Automation Magazine, vol. 13, no. 2, pp. 99-110, 2006.

[3] S. Thrun and M. Montemerlo, "The graph SLAM algorithm with applications to large-scale mapping of urban structures," The International Journal of Robotics Research, vol. 25, no. 5-6, pp. 403-429, 2006.
[4] J. Jr. Okamoto and V. C. Guizilini, "On-line SLAM using clustered landmarks with omnidirectional vision," Journal of the Brazilian Society of Mechanical Sciences and Engineering, vol. 32, pp. 468-476, 2010.

[5] O. Grasa, et al., "Visual SLAM for handheld monocular endoscope," IEEE Transactions on Medical Imaging, vol. 33, no. 1, pp. 135-146, 2014.

[6] T. Bailey and H. Durrant-Whyte, "Simultaneous localization and mapping (SLAM): Part II,” IEEE Robotics \& Automation Magazine, vol. 13, no. 3, pp. 108-117.

[7] G. Grisettiyz, C. Stachniss, and W. Burgard, "Improving grid-based slam with rao-blackwellized particle filters by adaptive proposals and selective resampling," in Proc. the 2005 IEEE International Conference on Robotics and Automation, 2005, ICRA 2005, IEEE.

[8] A. Diosi, G. Taylor, and L. Kleeman, "Interactive SLAM using laser and advanced sonar,” in Proc. the 2005 IEEE International Conference on Robotics and Automation, 2005, ICRA 2005, IEEE.

[9] M. Liu, S. Huang, and G. Dissanayake, "Feature based SLAM using laser sensor data with maximized information usage,” in Proc. 2011 IEEE International Conference on Robotics and Automation (ICRA), IEEE, 2011.

[10] C. Kerl, J. Sturm, and D. Cremers, "Dense visual SLAM for RGB-D cameras," in Proc. 2013 IEEE/RSJ International Conference on Intelligent Robots and Systems (IROS), IEEE, 2013.

[11] M. A. Markom, et al., "A mapping mobile robot using RP Lidar scanner," in Proc. 2015 IEEE International Symposium on Robotics and Intelligent Sensors (IRIS), IEEE, 2015.

[12] Z. Zhang, "Microsoft kinect sensor and its effect," IEEE Multimedia, vol. 19, no. 2, pp. 4-10, 2012

[13] Y. Quiñonez, et al., "Autonomous robot navigation based on pattern recognition techniques and artificial neural networks,” International Work-Conference on the Interplay Between Natural and Artificial Computation, Springer, Cham, 2015.

[14] G. Grisetti, C. Stachniss, and W. Burgard, "Improved techniques for grid mapping with rao-blackwellized particle filters,” IEEE Transactions on Robotics, vol. 23, no. 1, pp. 34-46, 2007

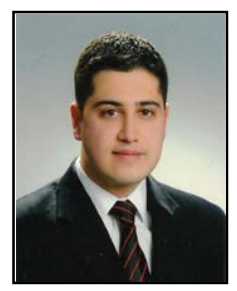

Mehmet Korkmaz received the B.Sc. and M.S. degrees from Department of Electrical and Electronics Engineering, Selcuk University, Konya, Turkey, in 2009 and 2013.

Mr. Korkmaz has been working as a researcher and $\mathrm{PhD}$ candidate in Department of Electrical and Electronics Engineering in Selçuk University, Konya. His main studies are fractional control theory and mobile robotics.

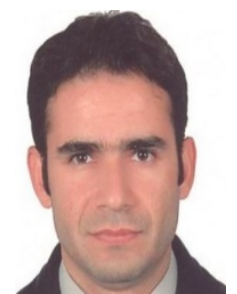

Akif Durdu has been an assistant professor with the Eletrical-Electronics Engineering Department at the Selcuk University (SU) since 2013. He earned a $\mathrm{PhD}$ degree in eletrical-electronics engineering from the Middle East Technical University (METU), in 2012. He received his B.Sc. degree in eletrical-electronics engineering in 2001 at the Selcuk University. His research interests include mechatronic design, search \& rescue robotics, robot manipulators, human-robot interaction, multi-robots networks and sensor networks. Dr. Durdu is teaching courses in control engineering, robotic and mechatronic systems

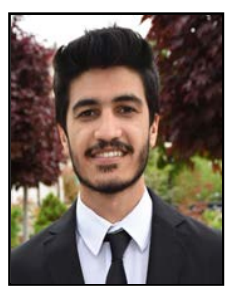

Yunus Emre Tusun received the B.Sc. degrees from Department of Electrical and Electronics Engineering, Selcuk University, Konya, Turkey, in 2017.

Mr. Tusun has been working as an electrical \& electronics engineer, Ankara. His main studies are mobile robotics and integrated circuits. 